\section{IHI ID 10 IMPROVED COMPLIANCE WITH EARLY SEPSIS MANAGEMENT IS ASSOCIATED WITH SHORTER HOSPITAL STAY}

'Larissa Rodriguez-Homs, 'Sabran Masoud, ${ }^{1}$ Oliver Jawitz, ${ }^{3}$ Harry Phillips III, ${ }^{3}$ Cara O'Brien, ${ }^{2}$ Paul Mosca. 'Duke University School of Medicine, USA; ${ }^{2}$ Duke University Department of Surgery, USA; ${ }^{3}$ Duke University Department of Medicine, USA

\subsection{6/ihisciabs. 10}

Background In 2018, Medicare began public reporting of hospital compliance with Sepsis and Septic Shock Early

\section{Number of Staffed Bed}

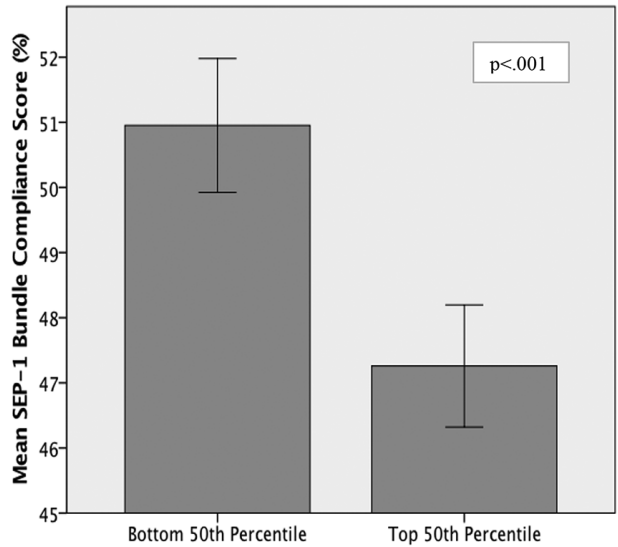

Number of Staffed Beds

Abstract IHI ID 10 Figure 1 SEP-1 bundle compliance scores by number of staffed beds. SEP-1 bundle compliance scores between hospitals with number of staffed beds in the bottom 50th percentile $(n=1498)$ and top 50th percentile $(n=1498)$. Smaller hospitals, measured by number of staffed beds, had statistically significant higher SEP-1 bundle compliance score $(50.95 \pm 20.29 \%)$ compared to larger hospitals $(47.26 \pm 18.50 \%), p<0.001$.

\section{A) Average Length of Stay}

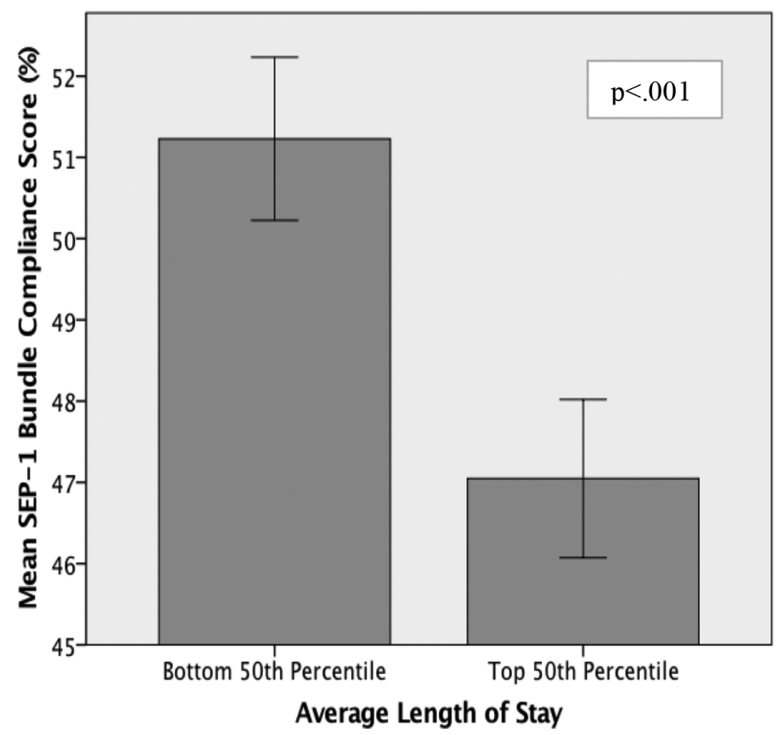

Management (SEP-1) bundle. We queried whether a relationship between SEP-1 bundle compliance, hospital size, and global measures of quality exists.

Objectives To determine the relationship between SEP-1 bundle compliance and hospital size, composite complication rate (PSI-90), average length of stay (ALOS) and hospital readmission rate.

Methods Data was obtained from Medicares Hospital Compare online database and The American Hospital Directory.

Composite Complication Rate

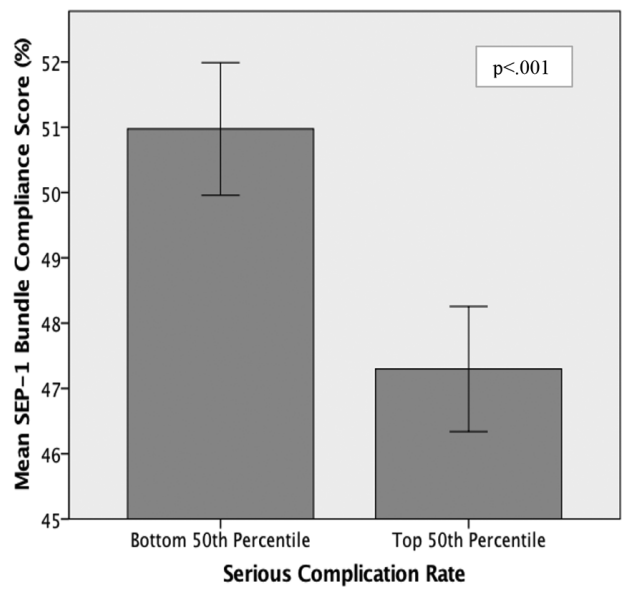

Abstract IHI ID 10 Figure 2 SEP-1 bundle compliance scores by composite complication rate. SEP-1 bundle compliance scores between hospitals with composite complication rates in the bottom 50th percentile $(n=1463)$ and top 50th percentile $(n=1371)$. Hospitals with lower composite complication rates had statistically significant higher SEP-1 bundle compliance score $(50.97 \pm 19.80 \%)$ compared to hospitals with higher composite complication rates $(47.30 \pm 18.09 \%), p<0.001$

\section{B) SEP-1 Bundle Compliance Score}

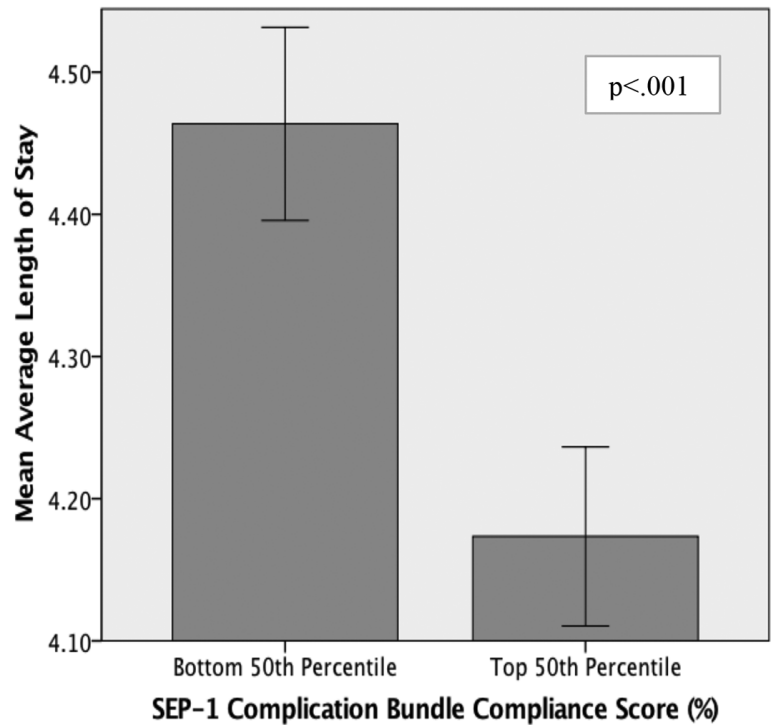

Abstract IHI ID 10 Figure 3 SEP-1 bundle compliance scores by average length of stay. A) SEP-1 bundle compliance scores between hospitals with average length of stay in the bottom 50th percentile $(n=1482)$ and top 50th percentile $(n=1483)$. Shorter average length of stay has statistically significant higher SEP-1 bundle compliance $(51.23 \pm 19.69 \%)$ compared to longer average length of stay $(47.05 \pm 19.14 \%), p<0.001$. B) Average length of hospital stay between SEP-1 bundle compliance scores in the bottom 50th percentile $(n=1484)$ and top 50th percentile $(n=1481)$. Lower SEP-1 bundle compliance score has statistically significant longer average length of stay (4.46 \pm 1.33 days) compared to shorter average length of stay (4.17 \pm 1.23 days), $p<0.001$ 
Abstract IHI ID 10 Table 1 Pearson correlations of SEP-1 bundle compliance versus hospital complications and quality, controlling for number of staffed beds

\begin{tabular}{|c|c|c|c|}
\hline \multirow[t]{2}{*}{ Control Variables } & \multirow[t]{2}{*}{ Variables } & \multicolumn{2}{|c|}{ Hospital Sep-1 Bundle Score } \\
\hline & & Coefficient & P-Value \\
\hline & Composite Complication Rate & -0.103 & $<0.001$ \\
\hline \multirow[t]{3}{*}{ None } & Staffed Beds & -0.114 & $<0.001$ \\
\hline & Average Length of Stay & -0.118 & $<0.001$ \\
\hline & Readmission Rate & -0.013 & 0.480 \\
\hline \multirow[t]{2}{*}{ Staffed Beds } & Composite Complication Rate & -0.093 & $<0.001$ \\
\hline & Average Length of Stay & -0.058 & 0.002 \\
\hline
\end{tabular}

Records missing SEP-1, complications, and discharge data were excluded. Pearson correlation, controlling for staffed beds, and an independent t-test were used for analysis.

Results A total of 2796 hospitals met inclusion criteria. An increased SEP-1 score was negatively associated with PSI-90 $(\mathrm{r}=-0.103, \mathrm{p}<0.001)$, staffed bed number $(\mathrm{r}=-0.114$, $\mathrm{p}<0.001)$, and ALOS $(\mathrm{r}=-0.118, \mathrm{p}<0.001)$. There was no association with 30 day readmission rate $(p=0.480)$. PSI-90 and ALOS remained significantly correlated with SEP-1 while adjusting for staffed beds. Figures 1 to 4 show independent t-test results relating SEP-1 scores to higher - and lower-performing hospitals on each of the indicated quality measures.

Conclusions Higher performance on SEP-1 compliance is associated with lower composite complication rate and shorter length of stay. The inverse relationship between SEP1 compliance and hospital size may reflect the challenges associated with implementation of new protocols in large, complex hospitals. While this study does not establish a causal relationship, the ability of hospitals to successfully implement SEP-1 may suggest a higher level of overall operational excellence.

\section{IHI ID 11 INTERPROFESSIONAL TEAM HUDDLE TO OPTIMIZE CARE DELIVERY TO PEDIATRIC INTENSIVE CARE UNIT (PICU) FAMILIES WITH COMPLEX PSYCHOSOCIAL STRESSORS}

Phoebe Yager, Sandra Clancy, Justine Romano. Massachusetts General Hospital, USA

\subsection{6/ihisciabs. 11}

Background Families with children admitted to the PICU experience complex emotional, psychological and financial stressors. Even thorough interprofessional teams can miss need identification or have duplicative efforts, compromising familycentered, high-quality care. In 2016, the interprofessional care team formally reviewed psychosocial stressors in $19 \%$ of PICU patients.

Objectives To increase PICU patients formally reviewed for psychosocial stressors by $30 \%$ over 12 months.

Methods The interprofessional team met to identify barriers to psychosocial care. Interventions included a time-limited, thriceweekly interprofessional huddle guided by a checklist. Process measures included frequency of huddles and member participation. Balance measures monitored time spent waiting for huddle. Performance over time was tracked with statistical process control (SPC) methodology. New interventions were introduced through PDSA cycles based on drivers of special cause variation.

Results PICU patients formally discussed by the interprofessional team increased from $19 \%$ to $90 \%$. Huddle frequency

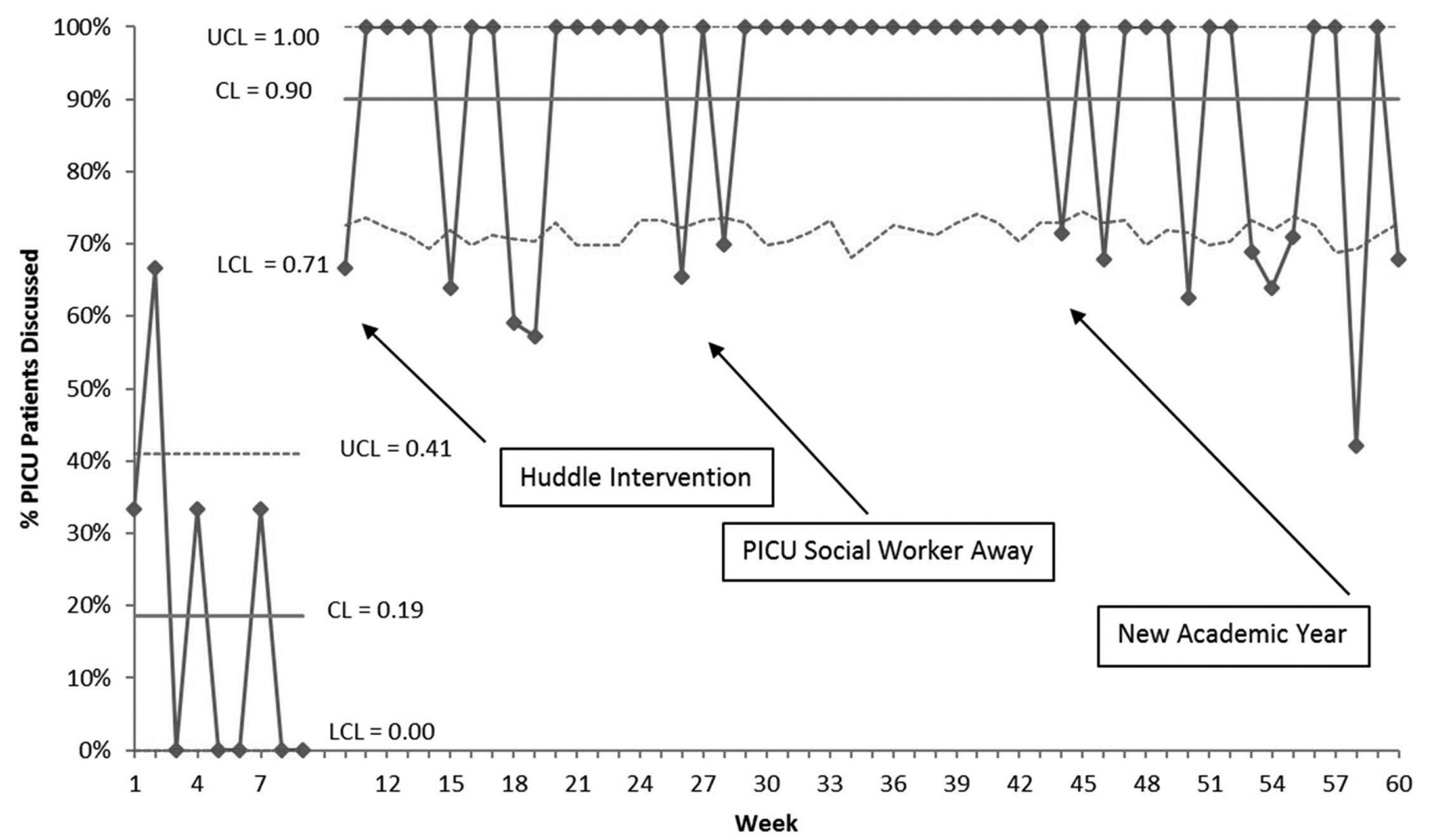

Abstract IHI ID 11 Figure 1 PICU phychosocial discussions by interprofessional team ( $p$ chart, 3 sigma, $n=1,511$ ) 